\title{
ReVIEW
}

\section{The essential adaptors of innate immune signaling}

\author{
Huihui Chen, Zhengfan Jiang \\ School of Life Sciences, Peking University, Beijing 100871, China \\ $\triangle$ Correspondence: jiangzf@pku.edu.cn \\ Received July 6, 2012 Accepted July 22, 2012
}

\begin{abstract}
Microbial components and the endogenous molecules released from damaged cells can stimulate germline-encoded pattern recognition receptors (PRRs) to transduce signals to the hub of the innate immune signaling network-the adaptor proteins MyD88/TRIF/ MAVS/STING/Caspase-1, where integrated signals relay to the relevant transcription factors IRF3/IRF7/NF-KB/ AP-1 and the signal transducer and activator of transcription 6 (STAT6) to trigger the expression of type I interferons and inflammatory cytokines or the assembly of inflammasomes. Most pleiotropic cytokines are secreted and bind to specific receptors, activating the signaling pathways including JAK-STAT for the proliferation, differentiation and functional capacity of immune cells. This review focuses on several critical adaptors in innate immune signaling cascades and recent progress in their molecular mechanisms.
\end{abstract}

\section{KEYWORDS innate immunity, adaptor, STING, STAT6}

\section{INTRODUCTION}

The innate immune response is initiated by germline-encoded pattern recognition receptors (PRRs) that serve as sensors to pathogen-associated molecular patterns (PAMPs) and damage-associated molecular patterns (DAMPs), providing the first line of defense against microbial infection or endogenous danger signals (Takeuchi and Akira, 2010). PAMPs refer to molecules released by pathogenic bacteria (e.g. lipopolysaccharides, LPS) or viruses (e.g. dsRNA/ ssRNA/dsDNA/ssDNA), whereas damaged or dying cells release endogenous molecules termed DAMPs (such as adenosine triphosphate, ATP; uric acid crystals and S100 proteins) that activate the immune system in a way analo- gous to PAMPs.

Currently identified PRRs include the membrane-bound Toll-like receptors (TLRs) and C-type lectin receptors (CLRs), as well as cytosolic retinoic acid-inducible gene I (RIG-I)-like receptors (RLRs), nucleotide-binding oligomerization domain (NOD)-like receptors (NLRs) and DNA sensors (e.g. DNAdependent activator of interferon regulatory factor, DAl; absent in melanoma-2, AIM2) (Takeuchi and Akira, 2010). PRRs have evolved in different cell types to detect PAMPs or DAMPs, including hematopoietic cells like dendritic cells (DCs) and macrophages as well as epithelial cells and fibroblasts. These receptors can recruit specific adaptor proteins, like myeloid differentiation primary response gene 88 (MyD88) or Toll/interleukin-1 receptor (TIR) domain-containing adaptor inducing IFN- $\beta$ (TRIF) in the TLR pathway, mitochondrial antiviral signaling protein (MAVS) downstream of RLRs, stimulator of interferon genes (STING) in the cytosolic DNA response pathway and, cysteine aspartic protease 1 (Caspase-1) as part of the inflammasome, all of which orchestrate the host innate responses, through activation of transcriptional factors such as nuclear factor $\mathrm{KB}(\mathrm{NF}-\mathrm{kB})$, activator protein 1 (AP-1) and interferon regulatory factors (IRFs), to trigger the production of type I interferons (IFNs), inflammatory cytokines and chemokines. Upon secretion, the cytokines function in an auto- or paracrine manner to induce the expression of hundreds of effecter genes involved in the generation of the innate anti-infection state (e.g. the Janus kinase (JAK)-signal transducer and activator of transcription (STAT) pathway activated by secreted type I interferons) (Brierley and Fish, 2005). Many of these innate immune-stimulated proteins can initiate adaptive immune responses involving antibody production and cytotoxic T-cell activity (Hebenstreit et al., 2006). It is amazing that such a complicated signal network is exquisitely regulated through only a few adaptors, which properly reflects the essential roles of these molecules. In this review, we summarize the 
signaling pathways mediated by such essential adaptors, and focus on the most recent findings regarding the interplay between STAT6 and the adaptor STING.

\section{MyD88-dependent signaling pathways}

MyD88 consists of a death domain (DD) and a TIR domain (Vogel et al., 2003). It is crucial for the proper responses of IL-1, IL-18 and nearly all TLRs except TLR3 in the activation of transcription factors NF-KB and AP-1 followed by the induction of proinflammatory genes. In addition, MyD88-dependent signaling downstream of TLR7 and TLR9 elicits the production of IFN- $\alpha$ and IFN- $\beta 1$ through the activation of IRF7 and/or IRF1 (Fig. 1) (Watters et al., 2007; Kumar et al., 2011).

In most cases, the receptors associate with MyD88 through homotypic interactions between their respective TIR domains, with the aid of TIR domain-containing adaptor protein (TIRAP)/MyD88 adaptor-like (Mal) in case of TLR2 and TLR4. This interaction allows MyD88 to recruit IL-1R-associated kinase (IRAK) family (IRAK4, IRAK1 and IRAK2) via DD interaction (Cao et al., 1996; Marta et al., 1997). IRAK1 is phosphorylated by IRAK4 and, after separated from MyD88, interacts with tumor necrosis factor (TNF)R-associated factor 6 (TRAF6), an E3 ubiquitin ligase that functions together with the Ubc13/Uev1A complex to generate dissociative lysine-63 $\left(\mathrm{K}^{63}\right)$-linked poly-ubiquitin chains (Horng et al., 2001). Unanchored $\mathrm{K}^{63}$ poly-ubiquitin chains bind and activate a complex comprising transforming growth factor $\beta$ (TGF- $\beta$ )-activated kinase 1 (TAK1) and TAK1-binding proteins (TAB1, -2 and -3) to phosphorylate inhibitor of kappaB kinase beta (IKBKB or IKK $\beta$ ) and mitogen-activated protein kinase kinase 6 (MAP2K6 or MKK6) (Wang et al., 2001). Then the IKK complex, made up of IKK- $\alpha$, IKK- $\beta$ and NF-KB essential modulator (NEMO), phosphorylates IKBa, leading to its degradation by the proteasome and the release of NF-KB, while phosphorylated MKK6 activates MAPKs including c-Jun N-terminal kinase 1/2 (JNK1/2), p38 and extracellular signal-regulated kinase $1 / 2$ (ERK1/2) to stimulate AP-1 activity. Active NF-KB and AP-1 translocate to the nucleus to turn on the expression of proinflammatory cytokines. Individually, TLR7 and TLR9 recruit MyD88 followed by the formation of a

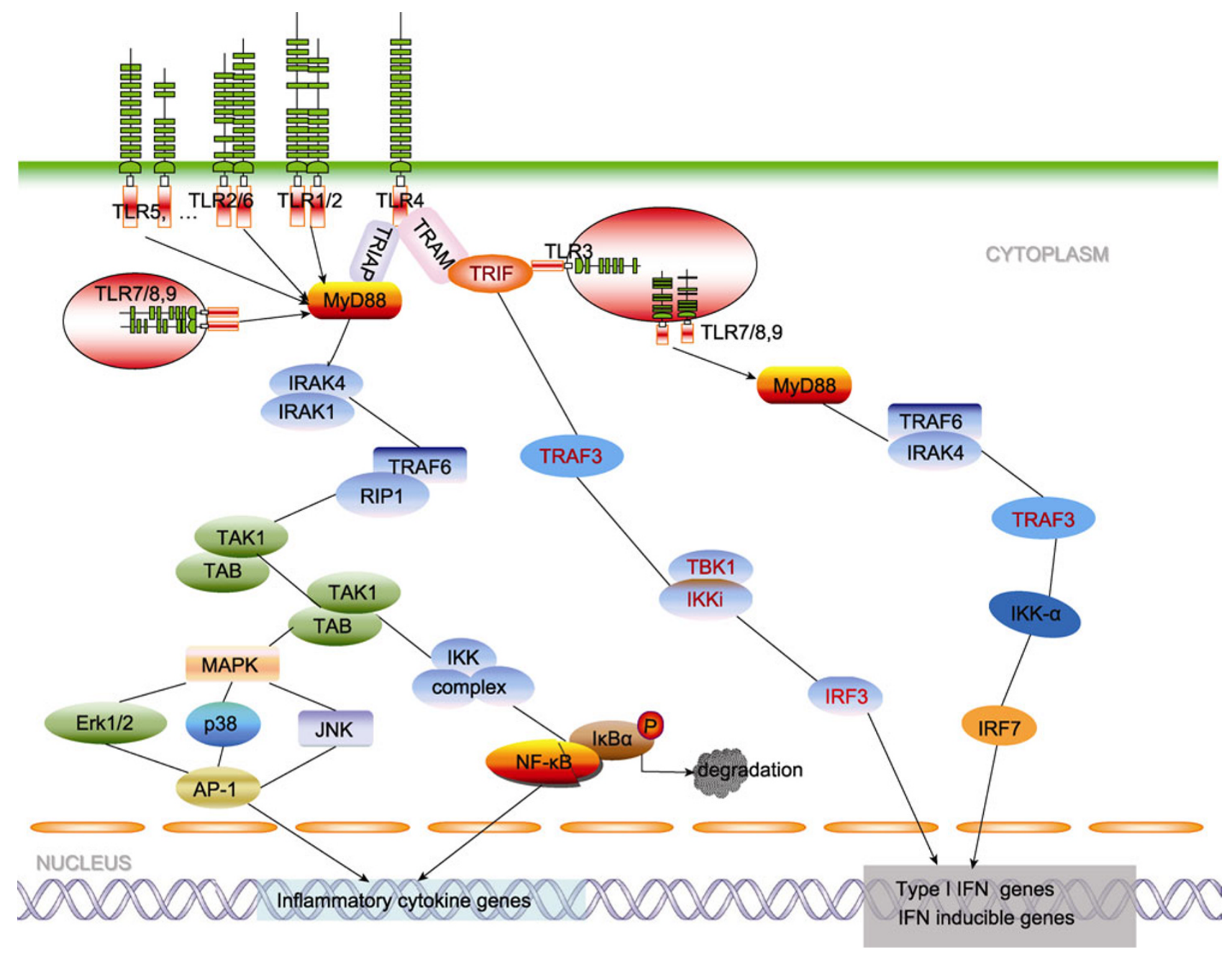

Figure 1. MyD88- and TRIF-dependent TLR signaling pathways. 
complex containing IRAK1, TRAF6, TRAF3, IKK- $\alpha$ and IRF7/IRF1, which results in the production of type I interferons (Brown et al., 2011). MyD88 also recruits IRF5 and triggers a TRAF6 signaling pathway that leads to the activation of NF-KB, where IRF4 competes with IRF5 for MyD88 binding (Chen, 2005; Lee and Kim et al., 2007).

\section{TRIF-dependent signaling pathways}

The fact that LPS and poly $(\mathrm{l}: \mathrm{C})$ (a viral dsRNA agonist) is still capable of activating NF-KB and MKK6 in MyD88-deficient cells indicates the existence of a MyD88-independent pathway (Fig. 1) (Kawai et al., 1999). In such contexts, TRIF/TIR domain-containing adaptor molecule 1 (TICAM-1) was found to be involved in the MyD88-independent responses. TRIF comprises 712 amino acids with three domains: a TRAF binding domain, a TIR domain and a C-terminal RHIM -receptor-interacting protein (RIP) homotypic interaction motif- domain (Yamamoto et al., 2003).

TLR3 directly interacts with TRIF, while TLR4 recruits TRIF through TRIF-related adaptor molecule (TRAM). The $\mathrm{N}$-terminus of TRIF interacts with TRAF3 and TRAF6 while its C-terminal RHIM domain recruits RIP1 and RIP3. As an E3 ubiquitin ligase, TRAF3 undergoes $\mathrm{K}^{63}$-linked self-ubiquitination to activate TRAF family member-associated NF-KB activator (TANK)-binding kinase-1 (TBK1) and/or inducible IKK (IKK-i)/IKK- $\varepsilon$. Then, phosphorylated TBK1 or IKK-i activates IRF3 and IRF7. The dimerization and nuclear translocation of IRF3 and IRF7 lead to the induction of type I interferons and IFN-stimulated genes (ISGs). TRAF6 activates NF-KB through multiple ways in complex with RIP1, TNFR-associated death domain protein (TRADD) and Fas-associated death domain protein (FADD). TRAF3 undergoes $\mathrm{K}^{48}$ - and $\mathrm{K}^{63}$-linked ubiquitination in either MyD88-dependent or TRIF-dependent signaling pathway, indicating its critical roles in both pathways, fine-tuning the production of proinflammatory cytokines and type I interferons ( Häcker et al., 2006; Tseng et al., 2010).

Signaling specificity of individual TLR can be imparted to downstream signaling molecules via the interaction of its TIR domain with MyD88, TRIF, TRAM or TIRAP. TLR stimulation can induce the production of proinflammatory cytokines, co-stimulatory molecules, type I interferons (IFN- $\alpha / \beta)$, type II interferon (IFN- $\gamma$ ) and chemokines. All TLRs except TLR3 recruit MyD88 to elicit inflammatory expression. TLR2 and TLR4 recruit TIRAP and MyD88 to activate NF-KB and MAPKs for the production of inflammatory cytokines, and TLR5 directly interacts with MyD88 to trigger NF-KB and MAPK signaling pathways. In addition, TLR4 (utilizes TRAM) and TLR3 activate IRF3 via TRIF for the induction of type I interferons. In plasmacytoid dendritic cells (pDCs), the TLR7/TLR9-MyD88 axis elicits the expression of IFN- $\alpha$ and IFN-related genes. MyD88-dependent signaling pathway: Upon specific ligand recognition, the TIR of TLR2 and TLR4 interacts with TIRAP and MyD88. Sequentially, MyD88 re- cruits and activates IRAK4, which in turn activates another IRAK family member-IRAK1. The downstream activation of TRAF6 by IRAK4/1 leads to the activation of TAK1 and TAB complex, which can stimulate both NF-KB and MAPK signaling pathways. Activated IKK complex (consisting of IKK- $\alpha$, IKK- $\beta$ and NEMO) causes the phosphorylation and degradation of $\mathrm{IKB} \alpha$, releasing NF-KB for its nuclear translocation and transcription initiation. In pDCs, MyD88 recruits IRAK4/1 to activate TRAF6 and ultimately the transcriptional factor IRF7. TRIF-dependent signaling pathway: Upon ligand binding, TLR3 directly recruits TRIF via its TIR domain while TLR4 utilizes TRAM to interact with TRIF. TRIF-recruited TRAF3 mediates the activation of TBK1 and/or IKK-i, which phosphorylate IRF3. IRF3 then dimerizes and translocates to the nucleus for the induction of cytokines such as type I interferons and IL-10. Meanwhile, TRIF also recruits TRAF6 and RIP1, which in turn activate the TAK1/TAB complex responsible for the activation of NF-kB.

\section{MAVS-dependent signaling pathways}

MAVS, also named interferon promoter stimulator-1 (IPS-1)/Caspase recruitment domain (CARD) adaptor inducing IFN- $\beta$ (Cardif) /virus-induced signaling adaptor (VISA) (Meylan et al., 2005; Kawai et al., 2005; Seth et al., 2005; Xu et al., 2005), is a mitochondrial outer membrane protein participating in the intracellular RLR pathways (Thompson and Locarnini, 2007). It is also implicated to locate to peroxisomes, and both localizations seem necessary for a maximal antiviral response. Peroxisomal MAVS activates IRF1 to induce the expression of ISGs as the primary antiviral responses, while mitochondrial MAVS works in a delayed stage for the vast production of ISGs and type I interferons via the activation of IRF3 (Dixit et al., 2010).

RLRs belong to the $D E x D / H$-box protein family, and serve as cytosolic RNA sensors. To date, there exist three members: RIG-I, melanoma differentiation associated factor 5 (Mda-5) and laboratory of genetics and physiology 2 (LGP2) (Yoneyama and Fujita, 2008). RIG-I and Mda-5 comprise of two N-terminal CARD domains, a DEAD-box helicase/ ATPase domain in the middle and a C-terminal regulatory domain. RIG-I mainly recognizes paramyxovirus such as Newcastle disease virus (NDV) and vesicular stomatitis virus (VSV). In addition, RIG-I recognizes Flaviviridae, such as Japanese Encephalitis virus (JEV) and hepatitis C virus (HCV). Meanwhile, RIG-I only senses relatively short dsRNA (about $1 \mathrm{~kb}$ ), and the shortest one (19-mer or 21-mer) ended with $5^{\prime}$ triphophorylation can stimulate host cells to induce massive production of type I interferons. However, chemosynthetic $5^{\prime}$ triphophorylated ssRNA has no effect on the upregulation of inflammatory cytokines or type I interferons, while dsRNA ended with 5 ' tri- or single phosphorylated, enable to activate the RIG-I signaling (Kato et al., 2006; Hornung et al., 2006). Picornavirus like encephalomyocarditis virus (EMCV) and Polio virus are largely recognized by 
Mda-5. Mda-5 prefers to sense long strand dsRNA (more than $2 \mathrm{~kb}$ ), and the intermediates of RNA virus replication in EMCV-infected cells including the higher-structured RNA consisted of dsRNA and ssRNA is able to activate Mda-5 signaling pathway. Moreover, both West Nile virus and dengue virus can activate RIG-I and Mda-5 signaling pathway simultaneously (Kato et al., 2008). LGP2, with no CARD, was originally recognized as a negative regulator for RIG-I and Mda-5 (Saito et al., 2007; Kato et al., 2008; Rothenfusser et al., 2012). Controversially, studies on $L G P 2^{-1-}$ mice and ATPase-inactivated mice unraveled a positive role for LGP2 in response to RNA virus, with the capability of promoting the interaction of RIG-I/Mda-5 with RNA (Satoh et al., 2010).

In RNA virus-invaded cells, RIG-I and Mda-5 experience conformational changes during RNA-binding and expose their CARDs to recruit MAVS, which activates TRAF3/TBK1/ IKK-i and then IRF3/IRF7, resulting in the expression of type I interferons. Meanwhile, MAVS induces NF-KB-dependent expression of proinflammatory factors by recruiting the TRADD/FADD/Caspase-8/Caspase-10 complex (Fig. 2).

During activation, RIG-I undergoes $\mathrm{K}^{63}$-linked ubiquitination on its CARD by tripartite motif containing 25 (TRIM25) and RNF135 (Zeng et al., 2010), whereas RNF125 downregulates $\mathrm{RIG}$-I signaling through $\mathrm{K}^{48}$-linked ubiquitination and degradation (Arimoto et al., 2007). The CARD domains of the RNA sensors RIG-I /Mda-5 bind to K63-polyubiquitin chains, and sequentially form the RIG-I or Mda-5 oligomers, which is essential for IRF3 activation and signaling regulation (Jiang et al., 2012). K63-polyubiquitinanted RIG-I oligomers are highly potent in aggregating and activating MAVS on the mitochondrial memberane through a prion-like manner, propagating the antiviral signaling cascade (Hou et al., 2011). According to a recent work, a Ser/Thr phosphatase eyes absent 4 (EYA4), is found to interact with MAVS and is es-

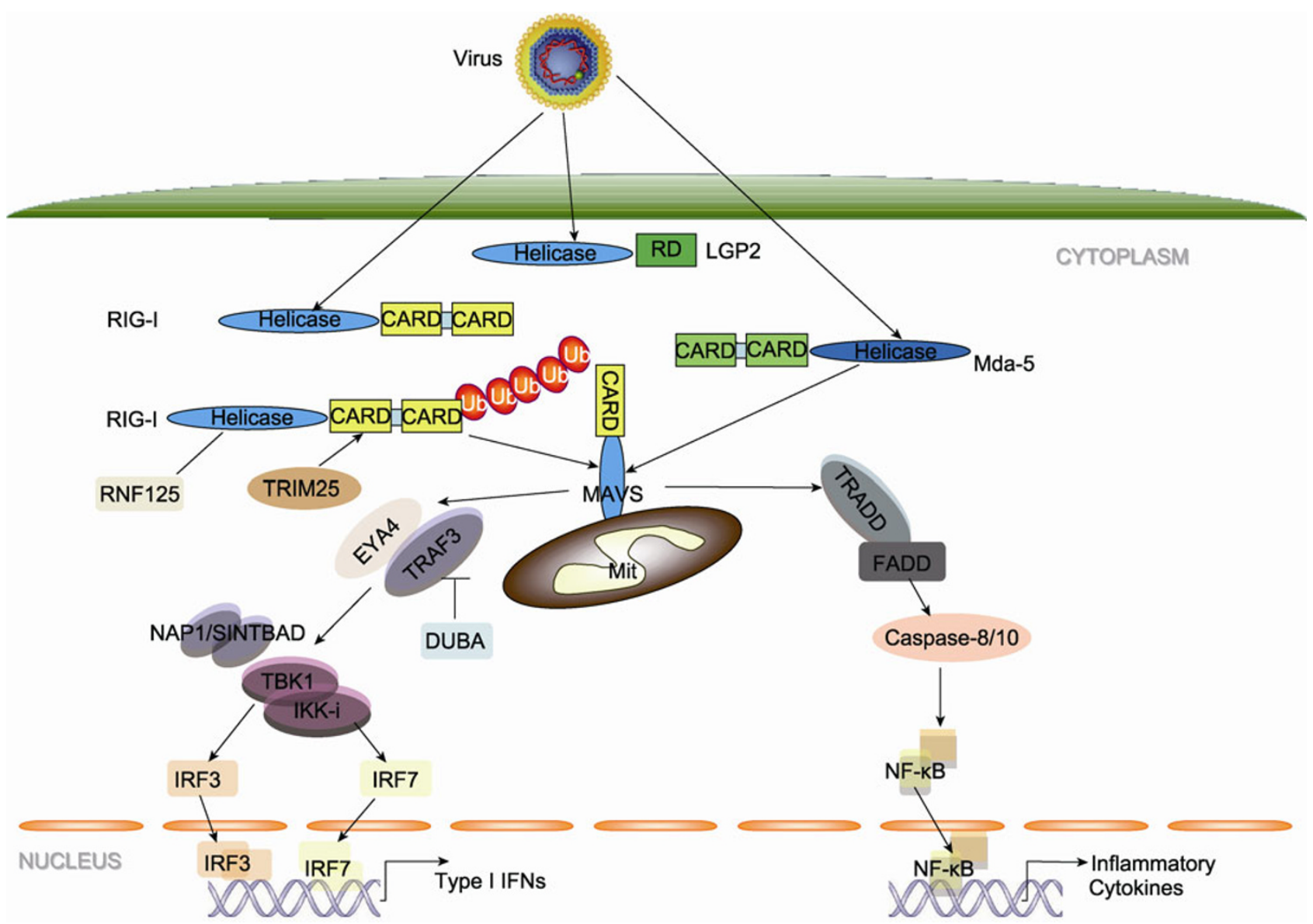

Figure 2. RLR-MAVS signaling pathways induced by RNA viruses. RIG-I and Mda-5 detect short dsRNA with 5' triphosphate ends and long dsRNA, respectively. By CARD domain interactions, they recruit MAVS and in turn activate IRF3/IRF7 through EYA4, TRAF3, NF-KB activating kinase-associated protein 1 (NAP1)/similar to NAP1 TBK1 adaptor (STINTBAD) and TBK1/IKK-i signaling cascades, leading to the expression of type I interferon genes. Meanwhile, MAVS activates NF-KB via the TRADD, FADD, Caspase-8/-10 signaling cascades, resulting in the production of proinflammatory cytokines. LGP2 functions as a positive regulator of RIG-I and Mda-5 signaling pathways. Activation of RIG-I is positively and negatively regulated by ubiquitin ligases TRIM25 and RNF125, respectively. RLR signaling requires polyubiquitination of TRAF3, which can be removed by a deubiquitinase, deubiquitinating enzyme $A$ (DUBA). 
sential for the production of IFN- $\beta$ (Okabe et al., 2009). A member of the NLR family, NLRX1 (or NOD9) colocalizes with MAVS at mitochondria and negatively regulates MAVS-dependent antiviral immune responses (Moore et al., 2008). Besides, the RLR pathway can be downregulated by autophagosome- related proteins such as autophagy-related gene 5 (ATG5) and ATG16L1. When mouse embryonic fibroblasts (MEFs) and conventional DCs (cDCs) lacking autophagosome are infected with RNA viruses, MAVS-resident mitochondria will dysfunction and produce massive reactive oxygen species (ROS), the release of which in turn triggers the induction of type I interferons.

\section{STING-dependent signaling pathways}

Stimulator of interferon genes STING, also named mediator of IRF3 activation (MITA)/endoplasmic reticulum (ER) interferon stimulator (ERIS)/membrane tetraspanner associated with MHC class II (MPYS) that locates to ER or/and mitochondria, is an important adaptor with a potent ability to induce type I interferons, interleukins and other proinflammatory factors (Ishikawa and Barber, 2008; Zhong et al., 2008; Sun et al., 2009). Homo sapiens STING consists of 379 amino acids, with a signal peptide and four transmembrane domains at its $\mathrm{N}$-terminus. In the vicinity of these domains there exist two ER retention sequences, $R^{76} I R^{78}$ and $R^{178} Y R^{180}$, which are essential for the proper localization and stability of STING (Sun et al., 2009).

STING-deficient mice are highly sensitive to both RNA viruses, e.g. vesicular stomatitis virus (VSV) and Sendai virus (SeV) and DNA viruses, e.g. herpes simplex virus type I (HSV-1), underscoring the physiological function of STING in antiviral immune responses (Ishikawa et al., 2009). However, STING cannot bind directly to RNA or DNA (Barber, 2011). In RNA-triggered pathway, STING is mainly involved in RIG-I rather than Mda-5 signaling. Our published data show that STING interacts with RIG-I but not Mda-5, consistent with the fact that STING-deficiency seldom affects poly $(\mathrm{I}: \mathrm{C})$ (recognized by Mda-5)-mediated signaling (Ishikawa et al., 2009). On the other hand, besides IFI16 and DDX41, cytosolic DNA sensor(s) that utilize STING for the induction of type I interferons remain to be identified or validated.

Intracellular localization is a primary factor for the function of STING, since it is transported into ER lumen from ER-bond ribosomes after translation, depending on the interaction between STING and a four-subnuit ( $\alpha-\Delta)$ complex, the translocon associated protein (TRAP) complex SSR2/TRAP $\beta$. Individually, the TRAP complex can bind with translocons, a three-subunit component comprising SEC61 $\alpha / S E C 61 \beta /$ SEC61y required for protein folding and secretion. As part of the exocyst component, Sec5 is found to colocalize with STING, essential for its transportation from post-Golgi to plasma membrane. In virus-infected cells, STING translocates from ER to Golgi, and then to cytoplasmic punctate structures, where it recruits TBK1 in the aid of RalB GTPase and Sec5. IRF3/IRF7 is thus activated to induce the expression of type I interferons. Importantly, STAT6 is also activated to trigger the production of chemokines, which is discussed in details later (Fig. 3).

The regulation of STING turns out very complicated. STING can be ubiquitinated and phosphorylated, and under the stimulation of SeV or poly (I:C)/poly dAdT, STING undergoes apparent oligomerization (Sun et al., 2009). And in vitro, STING interacts with TBK1 and IRF3, and stimulates the phosphorylation of IRF3 by TBK1 in the cytosolic DNA signaling pathway (Tanaka and Chen, 2012). A research claims that there is colocalization between autophagy-related gene 9a (ATG9a) and STING that negatively regulates STING signaling. In addition, 3' repairing exonuclease 1 (Trex1) plausibly downregulates STING-mediated signal pathways, for reverse transcribed human immunodeficiency virus type I (HIV-1) DNA activates STING to suppress its own replication or to eliminate other DNA viruses (Yan et al., 2011). Interestingly, STING is found to share homology with proteins of Flavivirus, such as dengue virus, yellow fever virus and Hepatitis C virus, all of which localize to ER (Ishikawa et al., 2009). Current evidence shows that human coronavirus (HcoV) NL63 and severe acute respiratory syndrome (SARS) CoV papain-like protease (PLP) interact with STING to negatively regulate signaling by disrupting the STING dimmers and preventing the ubiquitination of RIG-I/TBK1/IRF3 (Sun et al., 2012). It is yet-to-be validated if there are other viruses that inhibit this pathway to escape the host immune responses. Undoubtedly, such work would provide insights into therapeutic approaches of autoimmune diseases.

\section{Caspase-1-dependent signaling pathways}

The inflammasome is a multimeric danger-sensing platform that mainly exists in macrophages and dendritic cells. Activation of the inflammasome initiates the secretion of IL-1 $1 / / L-18$ and induces a Caspase-1-dependent cell death named "pyroptosis" to protect the host against pathogens (Schroder and Tschopp, 2010). Most inflammasomes are composed of NLRs or pyrin and hematopoletic IFN-inducible nuclear proteins with 200 amino acids (HIN-200) domain (PYHIN) family members and the protease Caspase- 1 (Schroder and Tschopp, 2010).

The NLRs is a large receptor family characterized by $\mathrm{N}$-terminal CARD or pyrin domains (PYD), a central nucleotide-binding and oligomerization domain(NOD), and C-terminal leucine-rich repeats (LRRs) responsible for ligand sensing. To date, three groups of the NLR family: NLR family PYD-containing 1 (NLRP1), NLRP3 and NLR family CARDcontaining 4 (NLRC4) are found to be involved in the formation of inflammasomes (Kanneganti, 2010).

NLRP3 inflammasome has been studied most extensively. Numerous PAMPs, DAMPs and several environment stimulating factors can activate the NLRP3 inflammasome. The 


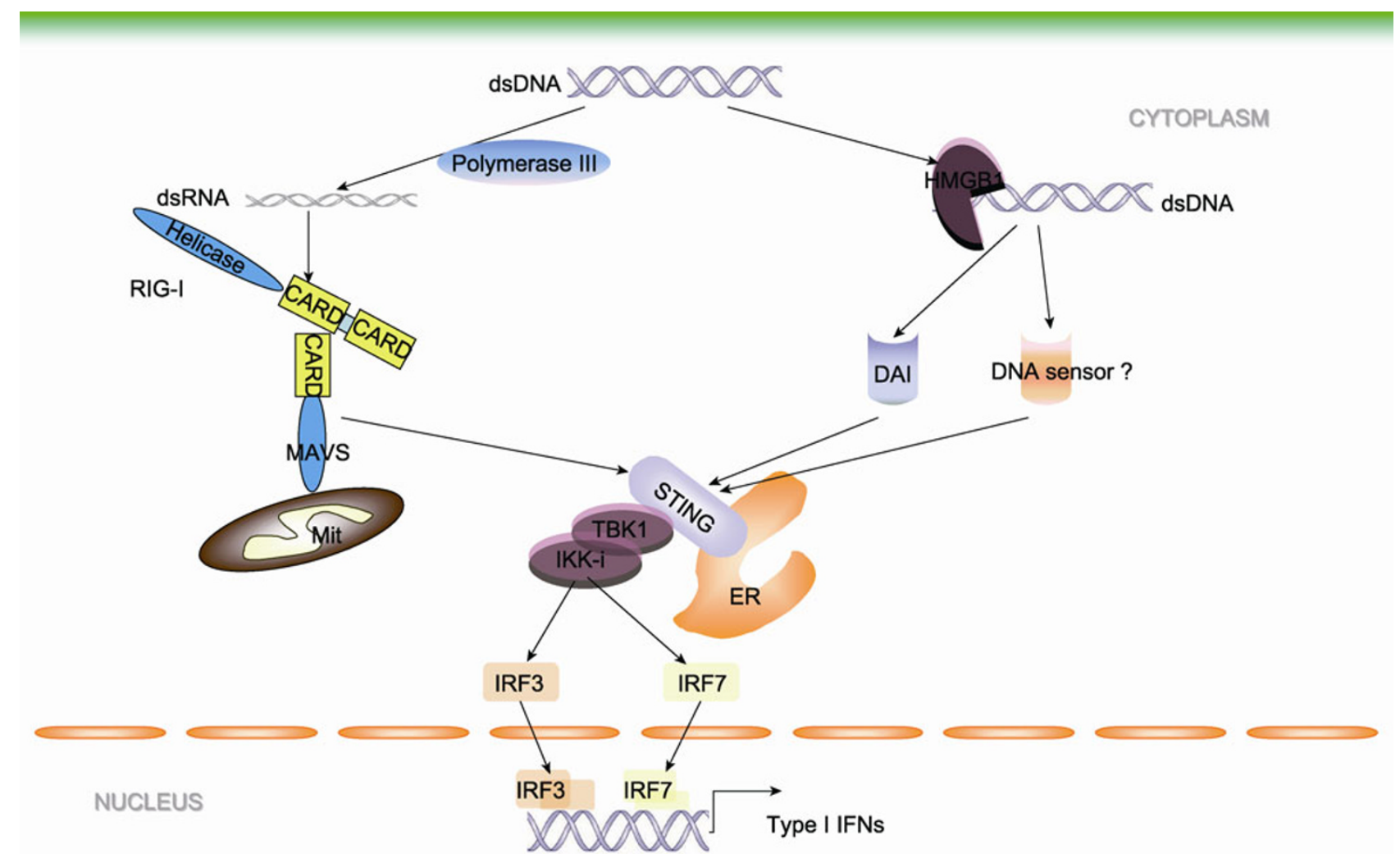

Figure 3. STING-mediated DNA sensing pathways. DNA viruses, bacteria genomic DNA and unprocessed endogenous DNA can be captured by high-mobility group protein B1 (HMGB1) and then recognized by DAl or unidentified DNA sensor(s). Alternatively, dsDNA is transcribed into dsRNA by polymerase III and recognized by RIG-I to induce type I interferons. Upon DNA stimulation, STING translocates from ER membrane to the cytoplasmic punctate structure, where it interacts with TBK1 and activates IRF3/IRF7.

oligomerization of activated NLRP3 enables the PYD domains coupling together and recruit ASC sequentially. The CARD domain of ASC then recruits pro-Caspase- 1 which in turn self-cleave to form the activated Caspase-1, namely p10/p20 tetramer, which consequently cut pro-IL-1 $\beta$ into its mature form. Recent work suggests that NLRC5 also participates in the formation of NLRP3 inflammasome, because bacteria-induced NLRP3 inflammasome is inhibited when silencing NLRP5 (Fig. 4) (Pétrilli et al., 2007).

NLRC4 (or IPAF) inflammasome is mainly comprised of NLRP4 and pro-Caspase-1, and is activated via direct interaction with the CARDs of Caspase-1 and NLRP4 to induce Caspase-1-dependent pyroptosis. The bacterial Type III secretion systems (T3SS) (such as Salmonella typhimuurium, Listeria, Francisella or Shigella flexneri) is essential for the activation of NLRC4 inflammasome (Zhao et al., 2011; Kofoed and Vance, 2011).

NLRP1 is the first receptor in NLR family that is found to be involved in inflammasome assembly and Caspase-1 activation. Homo sapiens NLRP1 inflammasome is composed of NLRP1, ASC, Caspase-1 and Caspase-5, which mainly recognizes muramyl dipeptide (MDP) deriving from bacterial peptidoglycan. As the only NLR receptor containing an extra C-terminal CARD domain, NLRP1 can recruit pro-Caspase-1 directly, but ASC can upregulate the activation of human NLRP1 inflammasome. Murine NLRP1 has three homology proteins: NLRP1a, NLRP1b and NLRP1c, among which, NLRP1b can be activated by lethal factor (LF) from anthrax toxin. Hence, it is still contorversial whether MDP can activate NLRP1 directly, and it is reported that NOD2 (NLRC2) serves as the intracellular receptor for MDP and participates in NLRP1 inflammasome assembly.

The PYHIN family comprises of four members: IFNinducible protein $X$ (IFIX), IFI16, myeloid nuclear differentiation antigen (MNDA) and AIM2, and their C-terminal HIN domain is in charge of sensing DNA and $\mathrm{N}$-terminal PYD domain recruits downstream adaptor ASC (Bürckstümmer et al., 2009; Fernandes-Alnemri et al., 2009; Hornung et al., 2009; Roberts et al., 2009.) AIM2 acts as a intracellular receptor for alien or endogenous dsDNA to induce Caspase-1-dependent maturation of IL-1 $1 \beta / I L-18$ and cell death like pyroptosis. The immune functions of other HIN-200 family members (MNDA, IFI16 and IFIX) remain unclear. 


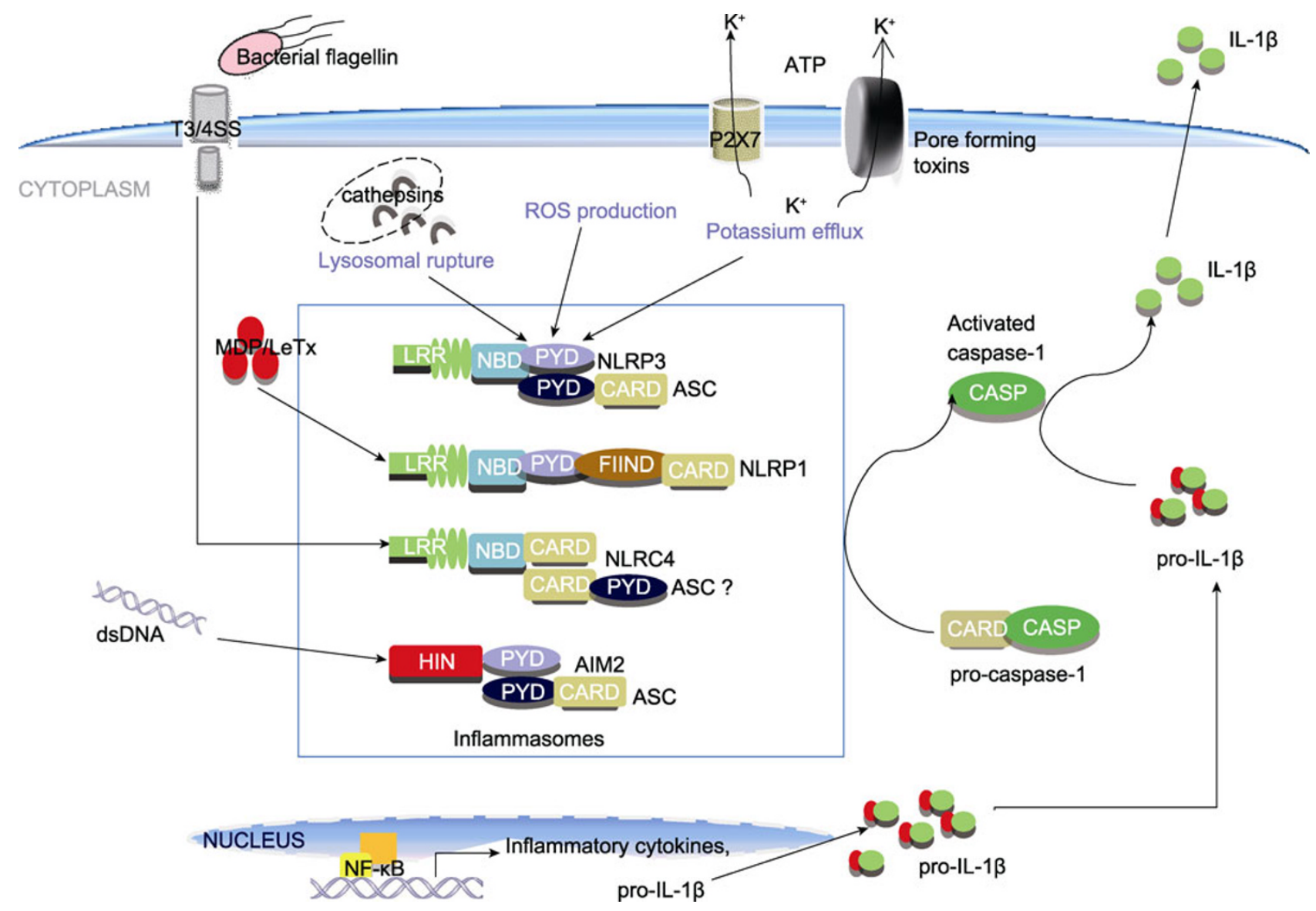

Figure 4. NLRP1、NLRP3、NLRC4 and AIM2 inflammasomes. NLRP1, NLRP3 and AIM2 recruit and activate Caspase-1 through the bridging molecule ASC, and the CARD domain of Caspase-1 is removed by autocleavage resulting in the formation of the activated Caspase-1 p10/20 tetramer. NLRC4 directly binds to Caspase-1 and induces the inflammasome assembly, yet there are several reports that stress the requirement for ASC in the NLRC4 inflammasome. Currently, there mainly exists three models for NLRP3 inflammasome activation: First, the NLRP3 agonist, ATP, triggers P2X7-dependent pore formation or pore forming toxins initiate the efflux of potassium, which directly elicit the assembly of NLRP3 inflammasome by a yet-unknown mechanism. Second, engulfed crystalline and the lysosomal protease cathepsin B plays an important role in the formation of NLRP3 inflammasome. Third, all of DAMPs and PAMPs, including ATP and crystalline activators can induce the formation of reactive oxygen species (ROS), which indirectly activate NLRP3 inflammasome by a yet-unidentified manner. Caspase-1 clustering induces autoactivation and caspase-1-dependent maturation and secretion of proinflammatory cytokines, such as IL-1 $\beta$.

\section{STAT6}

\section{Background information on STAT6}

STAT6 belongs to the Signal Transducer and Activator of Transcription family of proteins. In mammals, there are seven members: STAT1, $-2,-3,-4,-5 a,-5 b$ and -6 (Brierley and Fish, 2005). These proteins transmit signals from a receptor complex to the nucleus and activate gene expression, known as the classical JAK-STAT pathways. STAT6 mainly participates in IL-4/IL-13-mediated allergic reaction, playing a vital role in the differentiation of T-helper type 2 (Th2) cells (Hebenstreit et al., 2006; Chapoval et al., 2010).

The Homo sapiens STAT6 gene maps to chromosome 12q13.3-q14.1, while Mus musculus Stat6 gene lies clustered in the distal region of murine chromosome 10 . This protein is ubiquitously expressed, especially plentiful in B and T cells, and constantly active in most cell types. Human STAT6 consists of 847 amino acids (murine 837 amino acids), which specifies a size of $94 \mathrm{kDa}$ in an unmodified state. It contains an N-terminal $\alpha$-Helix domain, a DNA binding domain (DBD), a linker domain (LD), a Src homology $2(\mathrm{SH} 2)$ domain and a C-terminal transactivation domain (TAD) (Fig. 5) (Hebenstreit et al., 2006). The $\mathrm{N}$-terminus of STAT6 enriches $\alpha$-Helix motif, namely coiled-coil domain. Recent publications have demonstrated that the N-terminus of STATs is involved in its interaction with receptors, and the phosphorylation, nuclear translocation of STATs, However, it remains to be seen whether these findings have functional relevance to STAT6, as an isoform of STAT6 truncated at N-terminus appears to be fully functional. The DBD of STAT6 extends from posi- 
tions 268 to 430 , allowing DNA binding when STAT6 is present as a dimer. We previously reported that $\mathrm{Ser}^{407}$ in DBD is phosphorylated by TBK1 after virus infection, but the biological significance of this modification remains unclear (Chen et al., 2011). The SH2 domain of STAT6 has been shown essential for its binding to phosphorylated tyrosine residues on the cytoplasmic tail of the receptor and its own dimerization, followed by the reciprocal interaction of the $\mathrm{SH} 2$ domain with a phosphorylated tyrosine at position 641 . Our work suggested that when the $\mathrm{L}^{551}$ in $\mathrm{SH} 2$ is mutated, STAT6 can still be activated by IL-4 but not virus infection. STAT6 has the longest TAD among STAT family members, which is 147 amino acids in length and is rich of proline residues. STAT6 displays a dominant-negative feature without TAD. $S^{756}$ in TAD can be phosphorylated after IL-4 stimulation, but further studies are needed to unravel its functional significance.

\section{STAT6 in adaptive immunity}

The canonical IL-4/IL-13 signaling pathways

STAT6 is classically involved in the IL-4/IL-13 signaling (Fig. 6 right). The cytokines IL-4/IL-13 are produced by Th2 cells, mast cells and basophils. These two cytokines share similar physiological functions and are implicated in pathological conditions such as asthma, allergy and autoimmune diseases. Nevertheless, IL-4 is more active regulating Th2 cells development, whereas IL-13 is more involved in the regulation on respiratory hypersensitivity, mucus hypersecretion and Th2-type inflammation of the bowel. The receptors for IL-4 and IL-13 share several features including four conserved cysteine residues, a WSXWS motif, fibronectin type III modules in the extracellular domain and proline-rich box regions in the intracellular domain, which are critical for the binding to tyrosine kinases of JAK family. IL-4 receptors consist of two transmembrane proteins. In hematopoietic cells, the IL-4Ra chain binds to a common gamma chain $(y C)$ to form the type I IL-4R receptor. Most notably, IL-4Ra chain has high affinity with IL-4, and the $Y C$ chain is also a component of the receptors for IL-2, IL-7, IL-9, IL-15 and IL-21. In nonhematopoietic cells, the type II IL-4 receptor is formed by interaction of IL-4Ra with IL-13Ra1 instead of $\mathrm{YC}$ and recognizes both IL-4 and IL-13. IL-13 binds to IL-13Ra1 with higher affinity (Hebenstreit et al., 2006). In contrast, IL-13a2 has higher affinity for IL-13 but fails to induce a signal, indicating that IL-13a2 acts as a decoy receptor. The cytoplasmic tails of IL-4/IL-13 receptor subunits associate with tyrosine kinase of the Janus family: IL-4R $\alpha$ binds to JAK1, YC with JAK3 whereas IL-13Ra1 with JAK2 or TYK2.

Upon IL-4/IL-13 binding, heterodimerized receptors activate JAKs. Subsequently, JAKs phosphorylate three core tyrosine residues $\left(\mathrm{Y}^{575}, \mathrm{Y}^{603}\right.$ and $\left.\mathrm{Y}^{631}\right)$ in IL-4Ra, providing the docking sites for STAT6. Intriguingly, any one of the three tyrosine residues is competent to activate STAT6. Then JAKs further phosphorylate STAT6 at the receptor: activated STAT6 dimerizes through $\mathrm{SH} 2$ domain and translocates into nucleus to function as a transcriptional factor, regulating IL-4/IL-13-dependent signaling such as Th2 differentiation, immunoglobulin E (IgE) and chemokine production and mucus generation. STAT6 prefers sites with a 4-bp spacer (TTCNNNNGAA, the N4 site), which are mainly found in IL-4 responsive promoters.

It is notable that the activation of STAT6 by cytokines is very transient, peaking at $10-30 \mathrm{~min}$, and nearly undetectable after $4 \mathrm{~h}$, indicating a quick downregulating mechanism. It is reported that tyrosine phosphatase Shp-1 is involved in this dephosphorylating process. Other studies also indicate that STAT6 induces the expression of some negative regulators such as the suppressor of cytokine signaling (SOCS).

\section{Other STAT6-activating cytokines}

Besides IL-4/IL-13, many other cytokines have been reported to activate STAT6 in a similar way. IL-3 affects the proliferation of hematopoietic cells via tyrosine phosphorylation of STAT6. IL-15 activates STAT6 in different cells including T cell, NK cell, and mast cell to initiate the expression of anti-apoptosis genes like Bcl-xL. In some B cell lines, IFN- $\alpha$ was found to activate STAT6, which can even lead to the formation of STAT2/STAT6 heterodimer. In addition, platelet- derived growth factor (PDGF), a major mitogen and chemotactic factor for mesenchymal cells such as fibroblasts and smooth muscle cells, also exerts an effect on JAK-mediated STAT6 activation in NIH 3 T3 fibroblasts and 3T3-L1 preadipocytes. On the contrary, experiments using fibroblasts derived from STAT6 knockout mice do not support any dependence of STAT6 on PDGF. Researches on murine mastocytes demonstrate that IL-15 induces TYK2-mediated STAT6 activation consequently suppressing the apoptosis in mastocytes.

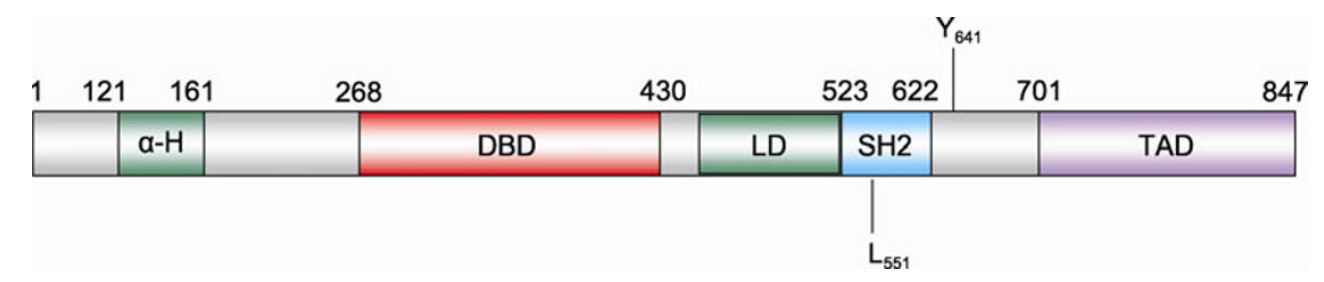

Figure 5. Domain structure of human STAT6. The protein has 847 aa and consists of five domains: $\alpha$-Helix domain, DBD (DNA binding domain), LD (linker domain), SH2 (Src homology 2 domain) and TAD (transactivation domain). 


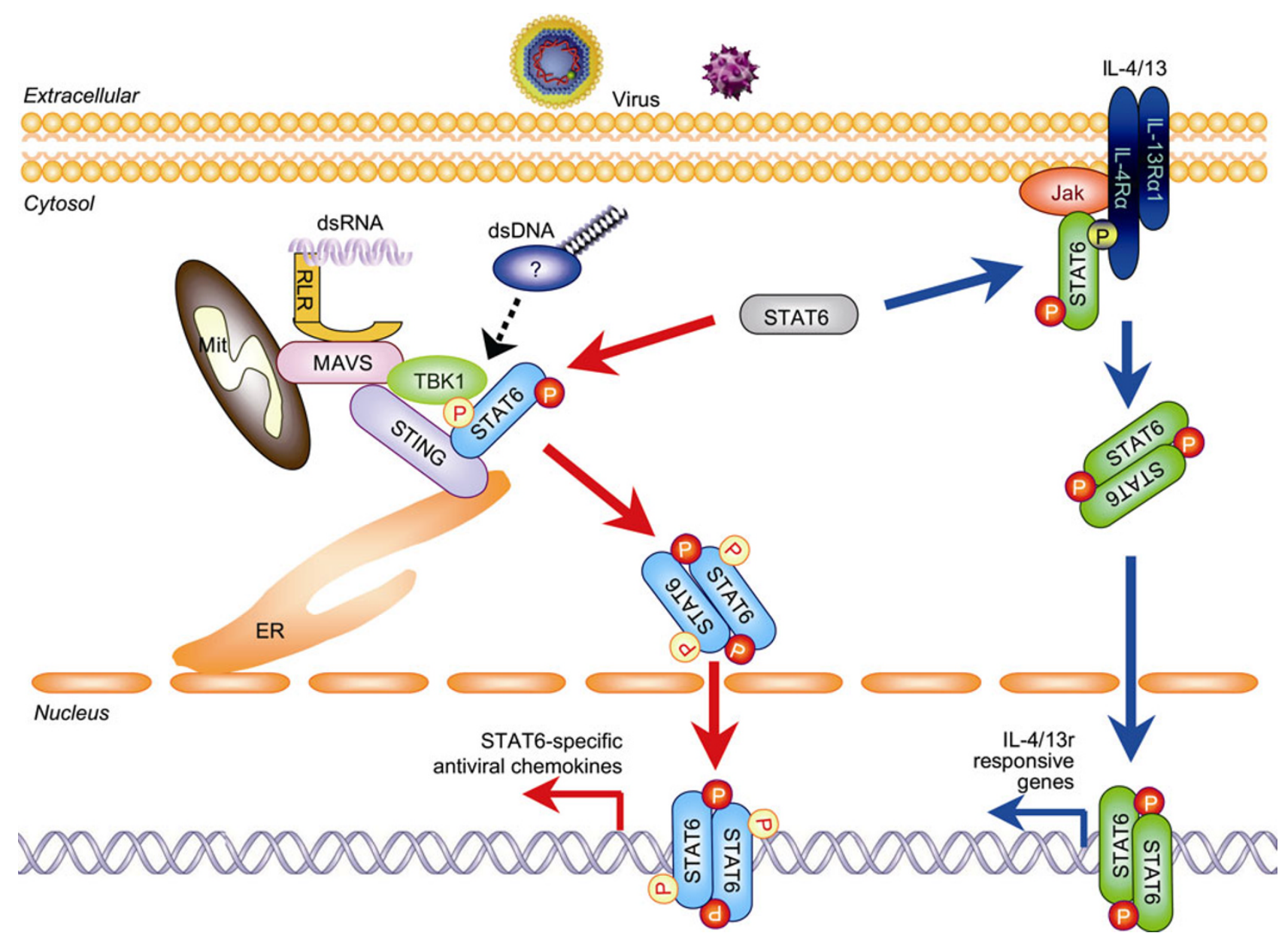

Figure 6. Right: The canonical IL-4/IL-13-STAT6 signaling pathways. Upon binding to IL-4/IL-13, JAKs located in the C terminal of IL-4Ra is phosphorylated and activated. Sequentially, JAKs phophorylate three tyrosine residues of the receptors, which provide docking sites for STAT6. Afterwards, STAT6 is phosphorylated by JAKs, leading to the dimerization and nuclear translocation to induce the expression of genes related to Th2 differentiation. Left: The STAT6-dependent antiviral signaling pathways. Upon DNA virus infection, activated STING recruits STAT6. TBK1 is also recruited by STING to phosphorylate STAT6 on $\mathrm{S}^{407}$, which in turn activates another unidentified tyrosine kinase to phosphorylate STAT6 on $\mathrm{Y}^{641}$, leading to the homodimerization and nuclear translocation of STAT6. STAT6 dimer then binds to its target sites to initiate transcription. On the other hand, RNA virus infection triggers STING activation through STING-MAVS interaction on MAVS-resident MAM or peroxisomal (Pex) membrane; activated STING then dissociates with MAVS and recruits STAT6 and TBK1, leading to STAT6 activation.

Moreover, in mast cells, kit ligand ( $\mathrm{KL})$, which is recognized by the receptor tyrosine kinase c-Kit, is implicated in hematopoiesis by activating STAT6 (Hundley et al., 2004). In cardiomyocytes, Angiotensin II (Ang II) activates JAK2 and STAT6 to regulate cardiovascular and renal homeostasis. Moreover, one leptin receptor obese receptor (OB-R) splice variant preferentially expressed in the hypothalamus codes for the activation of STAT6. Although these cytokines/factors showed to activate STAT6 in specific types of cells, the physiological relevance of these activation need to be further verified.

\section{STAT6 in innate immunity}

Previous studies suggest that STAT6 functions only in adap- tive immune responses, but our recent publication raised the possibility that STAT6 is also involved in antiviral innate immunity (Chen et al., 2011). We found that RNA and DNA viruses trigger the MAVS/STING/TBK1 axis, resulting in the expression of both type I interferons and STAT6-regulating genes, including chemokines like chemokine (C-C motif) ligand 2 (CCL2), CCL20 and CCL26 etc. Specifically, viruses or intracellular nucleic acids triggers STING to recruit STAT6 to endoplasmic reticulum, leading to the phosphorylation, dimerization and nuclear translocation of STAT6 for the induction of specific target genes responsible for immune cell homing. It was also found that activated STAT6 is phosphorylated at multiple sites, among which $\mathrm{Ser}^{407}$ is phosphorylated by TBK1 whilst $\mathrm{Tyr}^{641}$ is phosphorylated inde- 
pendent of JAKs, possibly by a thus unidentified tyrosine kinase. Importantly, the multi-site phosphorylation of STAT6 mentioned above is different from the classical IL-4/13 pathways. For instance, Leu ${ }^{551}$ is essential for STAT6 in response to virus infection, but it is not phosphorylated in activated STAT6 in the classical pathways. Stat $6^{--}$mice are remarkably susceptive to virus infection, and all these phenomena revealed the vital role of STAT6 in antiviral immune responses.

Since STAT6 is activated by a wide variety of cytokines, and also by viral infection, its physiological function is highly diversified. Previous work demonstrated that STAT6 performs its main role in mediating the biologic functions of IL-4/13, and therefore connects to the Th2 polarization of the immune system. It has been shown that more than 150 genes were direct targets of activated STAT6 and therefore up-regulated by IL-4/13 treatment, most of these genes are involved in human Th2 cell programming. STAT6 is also shown to be crucial in the development of protective immunity against gastrointestinal nematode parasites. Infection of mice with intestinal nematode parasites induces a strong Th2 cytokine response, thus triggers worm expulsion through an IL-4Ra-activated, Stat6-dependent mechanism (Hoeck and Woisetschläger, 2001).

Besides its role in Th2 cell development and immune response against nematode infection, studies from animal models and knockout mice demonstrated that STAT6 also actively functions in the pathogenesis of allergic diseases, like airway hyperresponsiveness (AHR), eosinophilic inflammation, or mucus production. Asthma is a chronic inflammatory disorder on remodeled airways, characterized by mast cell and eosinophil infiltration due to polarized Th2 response, leading to the abnormal secretion of cytokines like IL-3, $-4,-5,-9,-13$, and GM-CSF (Sehra et al., 2010). It is one of the top health problems, about three billion people are suffering from it worldwide. Recent studies on the pathogenic mechanism demonstrate that STAT6-dependent activation of Th2 response and the produced cytokines/chemokines is essential for the initiation of this chronic inflammation.

STAT6 deficiency also leads to impaired macrophage development and functional responses, including morphological changes and the decreased MHC class II expression and nitric oxide production. In addition, STAT6 has been found to be constitutively activated in transformed cell lines, suggesting that inappropriate activation of STAT6 is involved in uncontrolled cell growth in an oncogenic state. Human T-lymphotropic virus type I (HTLV-1) transformed cells constitutively activate the JAK-STAT6 pathway. However, solid evidence for the involvement of STAT6 in cellular transformation is still lacking.

Recent work found that STAT6 plays an important role in antiviral innate immunity. In response to virus infection, STAT6 was activated by STING-TBK1/IKKe. Activated STAT6 translocated to nuclei to regulate the expression of chemokines for homing of various immune cells including lymphocytes, monocytes, basophils, neutrophils, and eosinophils. The physiological function of STAT6 in this pathway was highlighted as Stat6 $^{-/-}$mice are sensitive to both DNA and RNA viral infections. Unlike other STAT6 activating pathways that are restricted to specific cell types, virus-induced STAT6 activation is found in all tested cell types, suggesting the fundamental importance of this signaling cascade (Chen et al., 2011).

Viral infection-activated STAT6 regulates the expression of chemotactic factors including CCL2, CCL20 and CCL26. These chemokines have been well documented for capable of attracting many types of cells including monocytes, macrophages, eosinophils, basophils, neutrophils and T cells, NK cells, and therefore implicated in the pathology of many diseases characterized by immune cell infiltration. CCL2 contributes to the sustained inflammation in chronic inflammatory disorders like atherosclerosis. It is required for initiating some autoimmune diseases (Mahad and Ransohoff, 2003), and functions in host defense against various pathogens (Winter et al., 2007). CCL20 could function as both an inflammatory and a homeostatic chemokine; its function in allergic airway diseases has been well documented (Weckmann et al., 2007). Recent work also suggested that CCL20 was implicated in the pathogenesis of psoriasis. CCL26 has multiple roles in allergic inflammation such as atopic dermatitis and asthma and also in the pathogenesis of eosinophilic esophagitis and Churg-Strauss syndrome (Bhattacharya et al., 2007). Since CX3CR1 is involved in Th1-mediated diseases such as rheumatoid arthritis, diabetes, lichen planus, and psoriasis, CCL26 is therefore likely involved in these diseases as well (Nakayama et al., 2010).

Given the fact that viral-infection-activated STAT6 regulates the expression of genes for various immune cells homing, the physiological function of STAT6 on this side, especially in development of the microbial-triggered allergic diseases, is just start to emerge.

\section{CONCLUDING REMARKS}

The past two decades have witnessed great progress in the understanding of molecular mechanisms underlying innate immune activation, providing important clues to how host cells recognize and resist the microbial harassment. Various pathogenic microorganisms invading the host can be identified by different PRRs, and through the innate immune signaling network, these diverse signals go to the corresponding hub molecules including MyD88, TRIF, MAVS, STING and Caspase-1, which drive the relevant downstream transcriptional factors IRF3/7, NF-kB, AP-1 and/or STAT6 to induce the expression of innate immune-related genes, leading to the elimination of the microbe and the activation of the adaptive immunity. Since PPRs, their relevant signaling adapters and downstream molecules locate in different cellular position, so it is of fundamental importance to elaborate how different signaling pathways were utilized in different cellular contexts. 
It is found that almost of the signaling molecules studied distributed differently, exemplified that the mRNA expression of 10 TLRs and 21 related genes performed quite variously in different tissues and cell lines (Masuhiro and Shinsaku, 2005). Besides, the influence of MyD88 on the gene expression during sepsis is strikingly organ-specific (Heike et al., 2006). The ability of PRRs to engage different adaptor proteins, crosstalk with other regulatory pathways and suppress or activate a plethora of kinases involved in a multitude of signaling pathways is an essential factor in shaping the type, strength, magnitude, timing and duration of inflammation and antiviral responses. This multidimensional signaling network precisely regulates both the innate and adaptive immune systems. Nevertheless, our knowledge of inflammation and antiviral biology is still in its infancy and many problems remain obscure. Specifically, the substantial antimicrobial mechanisms actually are beyond our understanding. Furthermore, we do not know much about the dynamic regulation of the entire immune system caused by crosstalk of multiple signaling cascades as well as the mechanisms fine-tuning the innate and adaptive immunity. Future investigations urgently need the introduction of more advanced and effective approaches, such as imaging techniques that can be used to define the dynamics of immune cell behavior and the system biology strategy to analyze and integrate accumulating knowledge (Chevrier et al., 2011). In future, these sophisticated methods will definitely enable scientists to indentify unknown PRRs exemplified the unidentified DNA sensors and uncover the initiation innate immunity and the integration of the signaling cascades. How to apply to use theoretical knowledge to clinic drug design and therapy for autoimmune diseases will be a huge challenge.

\section{ABBREVIATIONS}

YC, gamma chain; AHR, airway hyperresponsiveness; Ang II, Angiotensin II; AP-1, activator protein 1; ATG, autophagy-related gene; CARD, Caspase recruitment domain; Cardif, CARD adaptor inducing IFN- $\beta$; $C D C$, conventional DC; DBD, DNA binding domain; DC, dendritic cell; DUBA, deubiquitinating enzyme A; EMSV, encephalomyocarditis virus; ER, endoplasmic reticulum; ERIS, ER interferon stimulator; EYA4, eyes absent 4; FADD, Fas-associated death domain protein IFN, interferon; HcoV, human coronavirus; HCV, hepatitis C virus; HTLV-1. T-lymphotropic virus type I; HIV, human immunodeficiency virus; HMGB1, high-mobility group protein B1; HSV, herpes simplex virus type; IFIX, IFN- inducible protein X; Ig, immunoglobulin; IRAK, IL-1R-associated kinase; IRF, interferon regulatory factors; IPS-1, interferon promoter stimulator-1; ISG, IFN-stimulated gene; JEV, Japanese Encephalitis virus; KL, kit ligand; LD, linker domain; LF, lethal factor; LGP2, laboratory of genetics and physiology 2; LRR, leucine-rich repeats; MAP2K6 (MKK6), mitogen-activated protein kinase kinase 6; MAVS, mitochondrial antiviral signaling protein; MDP, muramyl dipeptide; MEF, mouse embryonic fibroblast; MNDA, myeloid nuclear differentiation antigen; MyD88, myeloid differentiation primary response gene 88; NDV, Newcastle disease virus; NEMO, NF-KB essential modulator;
NF-kB, nuclear factor $\mathrm{kB}$; NLRP1, NLR family PYD-containing 1; NLRC4, NLR family CARD-containing 4; NOD, nucleotide-binding and oligomerization; OB-R, obese receptor; $\mathrm{pDC}$, plasmacytoid $\mathrm{DC}$; pDGF, platelet- derived growth factor; PLP, papain-like protease; PRR, pattern recognition receptor; PYD, pyrin domain; RIP, RHIM-receptor-interacting protein; ROS, reactive oxygen species; SARS, severe acute respiratory syndrome; SeV, Sendai virus; STAT, signal transducer and activator of transcription; $\mathrm{SH} 2, \mathrm{Src}$ homology 2; SOCS, suppressor of cytokine signaling; STING, stimulator of interferon genes; T3SS, Type III secretion system; TAD, transactivation domain; TAK1, (TFG- $\beta$ )-activated kinase 1; TBK1, TRAF family member-associated NF-KB activator (TANK)-binding kinase; TGF- $\beta$, transforming growth factor $\beta$; TICAM-1, TRIF/TIR domain-containing adaptor molecule 1; TIR, Toll/interleukin-1 receptor; Th2, T-helper type 2; TNF, tumor necrosis factor; TRAM, TRIF-related adaptor molecule; TRADD, TNFR-associated death domain protein; TRAF6, (TNF)R-associated factor 6; TRAP, translocon associated protein; TRIF, TIR domain-containing adaptor inducing IFN- $\beta$; VISA, virus-induced signaling adaptor; VSV, vesicular stomatitis virus;

\section{REFERENCES}

Arimoto, K.I., Takahashi, H., Hishiki, T., Konishi, H., Fujita, T., and Shimotohno, K. (2007). Negative regulation of the RIG-I signaling by the ubiquitin ligase RNF125. Proc Natl Acad Sci U S A 104, 7500-7505.

Barber, G.N. (2011). Innate immune DNA sensing pathways: STING, AIMII and the regulation of interferon production and inflammatory responses. Curr Opin Immunol 23, 10-20.

Bhattacharya, B., Carlsten, J., Sabo, E., Kethu, S., Meitner, P., Tavares, R., Jakate, S., Mangray, S., Aswad, B., and Resnick, M.B. (2007). Increased expression of eotaxin-3 distinguishes between eosinophilic esophagitis and gastroesophageal reflux disease. Hum Pathol 38, 1744-1753.

Brierley, M.M., and Fish, E.N. (2005). Stats: multifaceted regulators of transcription. J Interf Cytok Res 25, 733-744.

Brown, J., Wang, H., Hajishengallis, G.N., and Martin, M. (2011). TLR-signaling networks: an integration of adaptor molecules, kinases, and cross-talk. J Dent Res 90, 417-427.

Bürckstümmer, T., Baumann, C., Blüml, S., Dixit, E., Dürnberger, G., Jahn, H., Planyavsky, M., Bilban, M., Colinge, J., Bennett, K. L., et al. (2009). An orthogonal proteomic-genomic screen identifies AIM2 as a cytoplasmic DNA sensor for the inflammasome. Nat Immunol 10, 266-272.

Cao, Z.D., Henzel, W.J., and Gao, X. (1996). IRAK: a kinase associated with the interleukin-1 receptor. Science 271, 1128-1131.

Chapoval, S., Dasgupta, P., Dorsey, N.J., and Keegan, A.D. (2010). Regulation of the Thelper cell type 2 (Th2)/T regulatory cell (Treg) balance by IL-4 and STAT6. J Leuk Biol 87, 1011-1018.

Chen, H.H., Sun, H., You, F.P., Sun, W.X., Zhou, X., Chen, L., Yang, J., Wang, Y.T., Tang, H., Guan, Y.K., et al. (2011). Activation of STAT6 by STING is critical for antiviral innate immunity. Cell 147, 436-446.

Chen, Z.J. (2005). Ubiquitin signalling in the NF-kB pathway. Nat Cell Biol 7, 758-765.

Chevrier, N., Mertins, P., Artyomov, M.N., Shalek, A.K., lannacone, M., Ciaccio, M.F., Gat-Viks, I., Tonti, E., DeGrace, M.M., Clauser, 
K.R., et al. (2011). Systematic discovery of TLR signaling components delineates viral-sensing circuits. Cell 147, 853-867.

Dixit, E., Boulant, S., Zhang, Y.J., Lee, A.S.Y., Odendall, C., Shum, B., Hacohen, N., Chen, Z.J., Whelan, S.P., Fransen, M., et al. (2010). Peroxisomes are signaling platforms for antiviral innate immunity. Cell 141, 668-681.

Fernandes-Alnemri, T., Yu, J.-W., Datta, P., Wu, J.H., and Alnemri, E.S. (2009). AIM2 activates the inflammasome and cell death in response to cytoplasmic DNA. Nature 458, 509-513.

Häcker, H., Redecke, V., Blagoev, B., Kratchmarova, I., Hsu, L.-C., Wang, G.G., Kamps, M.P., Raz, E., Wagner, H., Häcker, G., et al. (2006). Specificity in Toll-like receptor signalling through distinct effector functions of TRAF3 and TRAF6. Nature 439, 204-207.

Hebenstreit, D., Wirnsberger, G., Horejs-Hoeck, J., and Duschl, A. (2006). Signaling mechanisms, interaction partners, and target genes of STAT6. Cytokine Growth Factor Rev 17, 173-188.

Hoeck, J., and Woisetschläger, M. (2001). STAT6 mediates eotaxin-1 expression in IL-4 or TNF-alpha-induced fibroblasts. J Immunol 166, 4507-4515.

Horng, T., Barton, G.M., and Medzhitov, R. (2001). TIRAP: an adaptor molecule in the Toll signaling pathway. Nat Immunol 2, 835-841.

Hornung, V., Ablasser, A., Charrel-Dennis, M., Bauernfeind, F., Horvath, G., Caffrey, D.R., Latz, E., and Fitzgerald, K.A. (2009). AIM2 recognizes cytosolic dsDNA and forms a caspase-1-activating inflammasome with ASC. Nature 458, 514-518.

Hornung, V., Ellegast, J., Kim, S., Brzózka, K., Jung, A., Kato, H., Poeck, H., Akira, S., Conzelmann, K.-K., Schlee, M., et al. (2006). 5'-Triphosphate RNA is the ligand for RIG-I. Science 314, 994-997.

Hou, F.J., Sun, L.J., Zheng, H., Skaug, B., Jiang, Q.X., and Chen, Z.J. (2011). MAVS forms functional prion-like aggregates to activate and propagate antiviral innate immune response. Cell 146, 448-461.

Hundley, T.R., Gilfillan, A.M., Tkaczyk, C., Andrade, M.V., Metcalfe, D.D., Beaven, M.A. (2004). Kit and FcepsilonRI mediate unique and convergent signals for release of inflammatory mediators from human mast cells. Blood 104, 2410-2417.

Ishikawa, H., Ma, Z., and Barber, G.N. (2009). STING regulates intracellular DNA-mediated, type I interferon-dependent innate immunity. Nature 461, 788-792.

Jiang, X.M., Kinch, L.N., Brautigam, C.A., Chen, X., Du, F.H., Grishin, N.V., and Chen, Z.J. (2012). Ubiquitin-induced oligomerization of the RNA sensors RIG-I and Mda5 activates antiviral innate immune response. Immunity 36, 959-973.

Kato, H., Takeuchi, O., Sato, S., Yoneyama, M., Yamamoto, M., Matsui, K., Uematsu, S., Jung, A., Kawai, T., Ishii, K.J., et al. (2006). Differential roles of MDA5 and RIG-I helicases in the recognition of RNA viruses. Nature 441, 101-105.

Kawai, T., Adachi, O., Ogawa, T., Takeda, K., and Akira, S. (1999). Unresponsiveness of MyD88-deficient mice to endotoxin. Immunity $11,115-122$.

Kawai, T., Takahashi, K., Sato, S., Coban, C., Kumar, H., Kato, H., Ishii, K.J., Takeuchi, O., and Akira, S. (2005). IPS-1, an adaptor triggering RIG-I- and Mda5-mediated type I interferon induction. Nat Immunol 6, 981-988.

Kofoed, E.M., and Vance, R.E. (2011). Innate immune recognition of bacterial ligands by NAIPs determines inflammasome specificity. Nature 477, 592-595.

Kumar, H., Kawai, T., and Akira, S. (2011). Pathogen recognition by the innate immune system. Intl Rev Immunol 30, 16-34.

Lee, M.S., and Kim, Y.J. (2007). Signaling pathways downstream of pattern-recognition receptors and their cross talk. Annu Rev Biochem 76, 447-480.

Mahad, D.J., and Ransohoff, R.M. (2003). The role of MCP-1 (CCL2) and CCR2 in multiple sclerosis and experimental autoimmune encephalomyelitis (EAE). Semin Immunol 15, 23-32.

Marta, M., Jian, N., Ping, F., and Vishva, M.D. (1997). IRAK (Pelle) Family Member IRAK-2 AND MyD88 as Proximal Mediators of IL-1 Signaling. Science 278, 1612-1615.

Meylan, E., Curran, J., Hofmann, K., Moradpour, D., Binder, M., Bartenschlager, R., and Tschopp, J. (2005). Cardif is an adaptor protein in the RIG-I antiviral pathway and is targeted by hepatitis C virus. Nature 437, 1167-1172.

Moore, C.B., Bergstralh, D.T., Duncan, J.A., Lei, Y., Morrison, T.E., Zimmermann, A.G., Accavitti-Loper, M.A., Madden, V.J., Sun, L.J., Ye, Z.M., et al. (2008). NLRX1 is a regulator of mitochondrial antiviral immunity. Nature 451, 573-577.

Nakayama, T., Watanabe, Y., Oiso, N., Higuchi, T., Shigeta, A., Mizuguchi, N., Katou, F., Hashimoto, K., Kawada, A., and Yoshie, O. (2010). Eotaxin-3/CC chemokine ligand 26 is a functional ligand for CX3CR1. J Immunol 185, 6472-6479.

Nishimura, M., and Naito, S. (2005). Tissue-specific mRNA expression profiles of human toll-like receptors and related genes. Biol Pharm Bull 28, 886-892.

Okabe, Y., Sano, T., and Nagata, S. (2009). Regulation of the innate immune response by threonine-phosphatase of Eyes absent. Nature 460, 520-524.

Pétrilli, V., Papin, S., Dostert, C., Mayor, A., Martinon, F., and Tschopp, J. (2007). Activation of the NALP3 inflammasome is triggered by low intracellular potassium concentration. Cell Death Differ 14, 1583-1589.

Rothenfusser, S., Goutagny, N., Diperna, G., Monks, B.G., Schoenemeyer, A., Akira, S., Fitzgerald, K.A., Gong, M., and Yamamoto, M. (2005). The RNA helicase Lgp2 inhibits TLR-independent sensing of viral replication by retinoic acid-inducible gene-I. J Immunol 175, 5260-5268.

Saito, T., Hirai, R., Loo, Y.-M., Owen, D., Johnson, C.L., Sinha, S.C., Akira, S., Fujita, T., and Gale, M., Jr. (2007). Regulation of innate antiviral defenses through a shared repressor domain in RIG-I and LGP2. Proc Natl Acad Sci U S A 104, 582-587.

Satoh, T., Kato, H., Kumagai, Y., Yoneyama, M., Sato, S., Matsushita, K., Tsujimura, T., Fujita, T., Akira, S., and Takeuchi, O. (2010). LGP2 is a positive regulator of RIG-I- and MDA5-mediated antiviral responses. Proc Natl Acad Sci U S A 107, 1512-1517.

Schroder, K., and Tschopp, J. (2010). The inflammasomes. Cell 140, 821-832.

Sehra, S., Bruns, H.A., Ahyi, A.-N.N., Nguyen, E.T., Schmidt, N.W., Michels, E.G., von Bülow, G.-U., and Kaplan, M.H. (2008). IL-4 is a critical determinant in the generation of allergic inflammation initiated by a constitutively active Stat6. J Immunol 180, 3551-3559.

Sehra, S., Yao, Y.X., Howell, M.D., Nguyen, E.T., Kansas, G.S., Leung, D.Y.M., Travers, J.B., and Kaplan, M.H. (2010). IL-4 regulates skin homeostasis and the predisposition toward allergic 
skin inflammation. J Immunol 184, 3186-3190.

Seth, R.B., Sun, L., Ea, C.-K., and Chen, Z.J. (2005). Identification and characterization of MAVS, a mitochondrial antiviral signaling protein that activates NF-KB and IRF 3. Cell 122, 669-682.

Sun, L., Xing, Y.L., Chen, X.J., Zheng, Y., Yang, Y.D., Nichols, D.B., Clementz, M.A., Banach, B.S., Li, K., Baker, S.C., et al. (2012). Coronavirus papain-like proteases negatively regulate antiviral innate immune response through disruption of STING-mediated signaling. PLoS ONE 7, e30802.

Sun, W.X., Li, Y., Chen, L., Chen, H.H., You, F.P., Zhou, X., Zhou, Y., Zhai, Z.H., Chen, D.Y., and Jiang, Z.F. (2009). ERIS, an endoplasmic reticulum IFN stimulator, activates innate immune signaling through dimerization. Proc Natl Acad Sci U S A 106, 8653-8658.

Tanaka, Y., and Chen. Z.J. (2012). STING specifies IRF3 phosphorylation by TBK1 in the cytosolic DNA signaling pathway. Sci Signal 5, ra20.

Takeuchi, O., and Akira, S. (2010). Pattern recognition receptors and inflammation. Cell 140, 805-820.

Thompson, A.J.V., and Locarnini, S.A. (2007). Toll-like receptors, RIG-I-like RNA helicases and the antiviral innate immune response. Immunol Cell Biol 85, 435-445.

Tseng, P.-H., Matsuzawa, A., Zhang, W.Z., Mino, T., Vignali, D.A.A., and Karin, M. (2010). Different modes of ubiquitination of the adaptor TRAF3 selectively activate the expression of type I interferons and proinflammatory cytokines. Nat Immunol 11, 70-75.

Vogel, S.N., Fitzgerald, K.A., and Fenton, M.J. (2003). TLRs: differential adapter utilization by toll-like receptors mediates TLR-specific patterns of gene expression. Mol Interv 3, 466-477.

Wang, C., Deng, L., Hong, M., Akkaraju, G.R., Inoue, J.I., and Chen, Z.J. (2001). TAK1 is a ubiquitin-dependent kinase of MKK and IKK. Nature 412, 346-351.

Watters, T.M., Kenny, E.F., and O'Neill, L.A.J. (2007). Structure, function and regulation of the Toll/IL-1 receptor adaptor proteins. Immunol Cell Biol 85, 411-419.

Weckmann, M., Collison, A., Simpson, J.L., Kopp, M.V., Wark, P.A.B., Smyth, M.J., Yagita, H., Matthaei, K.I., Hansbro, N., Whitehead,
B., et al. (2007). Critical link between TRAIL and CCL20 for the activation of $\mathrm{TH} 2$ cells and the expression of allergic airway disease. Nat Med 13, 1308-1315.

Weighardt, H., Mages, J., Jusek, G., Kaiser-Moore, S., Lang, R., and Holzmann, B. (2006). Organ-secific role of MyD88 for gene regulation during polymicrobial peritonitis. Infect Immun 74, 3618-3632.

Winter, C., Taut, K., Srivastava, M., Länger, F., Mack, M., Briles, D.E., Paton, J.C., Maus, R., Welte, T., Gunn, M.D., et al. (2007). Lung-specific overexpression of CC chemokine ligand (CCL) 2 enhances the host defense to Streptococcus pneumoniae infection in mice: role of the CCL2-CCR2 axis. J Immunol 178, 5828-5838.

Xu, L.-G., Wang, Y.-Y., Han, K.-J., Li, L.-Y., Zhai, Z.H., and Shu, H.-B. (2005). VISA is an adapter protein required for virus-triggered IFN- $\beta$ signaling. Mol Cell 19, 727-740.

Yamamoto, M., Sato, S., Hemmi, H., Hoshino, K., Kaisho, T., Sanjo, H., Takeuchi, O., Sugiyama, M., Okabe, M., Takeda, K., et al. (2003). Role of adaptor TRIF in the MyD88-independent toll-like receptor signaling pathway. Science 301, 640-643.

Yan, N., Regalado-Magdos, A.D., Stiggelbout, B., Lee-kirsch, M.A., and Lieberman, J. (2011). The cytosolic exonuclease TREX1 inhibits the innate immune response to human immunodeficiency virus type 1. Nat Immunol 11, 1005-1013.

Yoneyama, M., and Fujita, T. (2008). Structural mechanism of RNA recognition by the RIG-I-like receptors. Immunity 29, 178-181.

Zeng, W.W., Sun, L.J., Jiang, X.M., Chen, X., Hou, F.J., Adhikari, A., $\mathrm{Xu}, \mathrm{M}$., and Chen, Z.J. (2010). Reconstitution of the RIG-I pathway reveals a signaling role of unanchored polyubiquitin chains in innate immunity. Cell 141, 315-330.

Zhao, Y., Yang, J.L., Shi, J.J., Gong, Y.-N., Lu, Q.H., Xu, H., Liu, L.P., and Shao, F. (2011). The NLRC4 inflammasome receptors for bacterial flagellin and type III secretion apparatus. Nature 477, 596-600.

Zhong, B., Yang, Y., Li, S., Wang, Y.-Y., Li, Y., Diao, F.C., Lei, C.Q., He, X., Zhang, L., Tien, P., et al. (2008). The adaptor protein MITA links virus-sensing receptors to IRF3 transcription factor activation. Immunity 29, 538-550. 